\title{
AN EXPLOSIVE INSTABILITY IN MAGNETIC FLUIDS
}

\author{
BY
}

\author{
S. K. MALIK AND M. SINGH
}

Simon Fraser University, Burnaby, British Columbia, Canada

\begin{abstract}
The stability of a magnetic fluid subject to a normal magnetic field at the second harmonic resonance is investigated. The stability characteristics are examined on the basis of two coupled dynamical equations that are solved numerically. The solution reveals that there exists an explosive instability when the applied magnetic field strength is in the neighbourhood of the bifurcation magnetic field strength $H_{\mathrm{m}}$.
\end{abstract}

1. Introduction. The normal field instability in ferrofluid flows has drawn considerable attention in recent years [1-9]. Cowley and Rosensweig [2] have demonstrated that an instability sets in when the applied magnetic field $H$ is normal to the fluid interface and is slightly greater than the critical magnetic field $H_{\mathrm{c}}$. The flat interface deforms to form a regular hexagonal pattern and the subsequent finite amplitude state is time independent. In such an instability, $H=H_{\mathrm{c}}$ is a point of bifurcation, and the system becomes unstable when $H \geq H_{\mathrm{c}}$. Gailitis [4] deduced the nature of transition in the normal field system and provided a global diagram of the surface peak amplitude against the magnetic field. The stability diagram exhibited hard transition and hysteresis in an increasing magnetic field. Another hard transition was obtained when the magnetic field was allowed to decrease. The analysis revealed that the surface of the magnetic field has three possible configurations of stable equilibrium: a flat surface, an array of hexagonal waves, and an array of square waves.

Using finite element computation, Boudouvis, et al. [7] confirmed the results obtained by Gailitis [4]. The theoretical investigations were in agreement with careful experimental measurements of free surface profiles. Nonlinear stability and bifurcation analysis predicted accurately experimentally observed first-order excitation with hysteresis.

With the Liapunov-Schmidt reduction procedure, Trombly and Thomas [5] introduced a change of coordinates that fixed the free boundary in the static ferrofluid equation. They demonstrated the existence of a nontrivial branch of the equilibrium solution that bifurcates from the trivial solution at the critical magnetic field strength. Silber and Knobloch [8] employed the singularity approach to extend the work of Trombly and Thomas [5] to study the stability of nontrivial states and the 
possibility of secondary bifurcation. The effect of temporal as well as spatial modulation on such an instability was studied by Malik and Singh [3]. Their analysis reveals that the evolution of the amplitude of this instability is governed by the nonlinear Klein-Gordon equation, which yields the solitary and kink solutions as special cases. Furthermore, Malik and Singh [3] and Kant and Malik [10] pointed out that when the normalized wavenumber $k$ equals $\left[\frac{1}{2}(\rho-1)\right]^{1 / 2}$, where $\rho$ denotes the ratio of the densities of the two fluids involved, the second- and third-order solutions become singular. The appearance of singularity or the existence of zero divisor is the manifestation of the second harmonic resonance phenomenon. In such a case, the fundamental mode and the second harmonic mode travel with the same phase speed.

In this presentation, we shall examine the phenomenon of second harmonic resonance for the critical stage of the instability in the neighbourhood of the critical magnetic field. Our analysis applies to the branch referred to as "abede" in the paper by Gailitis [4]. The effect of second harmonic resonance was not studied by earlier workers [1]-[9]. The solution we obtain exhibits explosive instability when the applied magnetic field strength is in the neighbourhood of the bifurcation magnetic field strength $H_{\mathrm{m}}$. We shall use the method of multiple scales. Such an analysis requires special scalings $[12,13]$, which are formulated in Sec. 3. We study the harmonic resonance when the frequencies and the wavenumbers of the two interacting waves, i.e., the fundamental and the second harmonic modes, satisfy the conditions $\omega_{2}=2 \omega_{1}$ and $k_{2}=2 k_{1}$.

In Sec. 4, we obtain the dynamical equations governing the amplitudes of the interacting waves. These nonlinear equations have eight degrees of freedom and, therefore, seem to be nonintegrable. Instead, we solve the system numerically as an initial value problem by using fourth-order Runge-Kutta method. The integration reveals that both the modes diverge at finite times, thus leading to the existence of an explosive instability which is dependent upon the parameters of the problem and the initial conditions.

2. Basic equations. We consider the normal magnetic field instability of two semiinfinite, incompressible, inviscid ferrofluids with densities $\rho_{1}$ and $\rho_{2}$, respectively. The gravitational force per unit mass is $\mathbf{g}(0,0,-1)$ whereas the magnetic field $\mathbf{H}(0,0,01)$ acts normal to the interface. The fluids with magnetic permeabilities $\mu_{1}$ and $\mu_{2}$ occupy the regions $z<0$ and $z>0$, respectively. The flow field is assumed to be irrotational. The velocity potential $\phi(\mathbf{V}=\nabla \phi)$ and the magnetic potential $\psi(\mathbf{H}=-\boldsymbol{\nabla} \psi)$ are given by

$$
\begin{array}{ll}
\nabla^{2} \phi^{(1)}=0=\nabla^{2} \psi^{(1)}, & -x<z<\eta(x, t), \\
\nabla^{2} \phi^{(2)}=0=\nabla^{2} \psi^{(2)}, & \eta(x, t)<z<x,
\end{array}
$$

where $\eta(x, t)$ is the elevation of the free surface from the unperturbed level. Since the motion must vanish away from the interface, we require

$$
\left|\nabla \phi^{(1)}\right| \rightarrow 0, \quad\left|\nabla \psi^{(1)}\right| \rightarrow 0 \quad \text { as } z \rightarrow-x
$$


and

$$
\left|\nabla \phi^{(2)}\right| \rightarrow 0, \quad\left|\nabla \psi^{(2)}\right| \rightarrow 0 \quad \text { as } z \rightarrow+\infty .
$$

At the free surface $z=\eta(x, t)$, the various boundary conditions are given by

$$
\begin{aligned}
\frac{\partial \eta}{\partial t}+\frac{\partial \phi^{(l)}}{\partial x} \cdot \frac{\partial \eta}{\partial x} & =\frac{\partial \phi^{(l)}}{\partial z} \quad(l=1,2) \\
\mu_{1} \mathbf{H}_{\mathrm{n}}^{(1)} & =\mu_{2} \mathbf{H}_{\mathrm{n}}^{(2)} \\
\mathbf{H}_{\mathrm{t}}^{(1)} & =\mathbf{H}_{\mathrm{t}}^{(2)}
\end{aligned}
$$

and

$$
\begin{aligned}
{\left[\rho_{1} \frac{\partial \phi^{(1)}}{\partial t}-\rho_{2} \frac{\partial \phi^{(2)}}{\partial t}\right.} & +\left(\rho_{1}-\rho_{2}\right) g \eta-T \frac{\partial^{2} \eta}{\partial x^{2}}\left(1+\left(\frac{\partial \eta}{\partial x}\right)^{2}\right)^{-3 / 2} \\
& +\frac{1}{2}\left(\left(\rho_{1}\left(\nabla \phi^{(1)}\right)^{2}-\rho_{2}\left(\nabla \phi^{(2)}\right)^{2}\right)\right] n_{i}+T_{i j}^{(1)} n_{j}=T_{i j}^{(2)} n_{j}
\end{aligned}
$$

where $T_{i j}^{(l)}$ represents the magnetic stress tensor

$$
T_{i j}^{(l)}=-\frac{\delta_{i j}}{4 \pi} \int_{0}^{H}\left[\mu-\rho\left(\frac{\partial \mu}{\partial \rho}\right)_{H, \theta}\right] \mathbf{H} d \mathbf{H}+\mu \frac{\mathbf{H H}}{4 \pi} .
$$

Here, $\mu=\mu(H, \theta, \rho)$, where $\theta$ denotes the temperature and $n$ the outward drawn normal. The quantities $H_{\mathrm{n}}, H_{\mathrm{t}}$, stand for the normal and the tangential components of the magnetic field, respectively, and $T$ is the coefficient of the surface tension. We assume the magnetic fluids to be linearly magnetizable and the flows to be isothermal. The superscripts in the various physical quantities refer to media 1 and 2.

3. Formulation of the problem. To describe the resonance interaction between two marginally unstable modes of small but fine amplitude waves, we employ the method of multiple scales formulated by Weissman [12] and Murakami [13]. Towards this goal, we introduce a small parameter $\varepsilon=\left(H^{2} / H_{\mathrm{m}}^{2}-1\right)^{1 / 2}$. This parameter defines the departure of the system from the bifurcation value $H_{\mathrm{m}}$, which we shall obtain later in Sec. 4. The difference between the applied magnetic field and its bifurcation value $H_{\mathrm{m}}$ is assumed to be small. We discuss the stability of the system in the neighbourhood of the neutral curve by letting the bifurcation parameter $H^{2}$ be $H_{\mathrm{m}}^{2}\left(1 \pm \varepsilon^{2}\right)$.

For significant interaction to occur at such a resonance, we introduce spatial and temporal scales $x_{n}=\varepsilon^{n} x, t_{n}=\varepsilon^{n} t$. To describe the nonlinear interactions in a slightly unstable region, we expand the various physical quantities as

$$
\begin{aligned}
& \phi(x, z, t)=\sum_{n=1}^{3} \varepsilon^{n+1} \phi_{n+1}+O\left(\varepsilon^{5}\right), \\
& \psi(x, z, t)=\sum_{n=1}^{3} \varepsilon^{n+1} \psi_{n+1}+O\left(\varepsilon^{5}\right),
\end{aligned}
$$




$$
\eta(x, t)=\sum_{n=1}^{3} \varepsilon^{n+1} \eta_{n+1}+O\left(\varepsilon^{5}\right)
$$

where $\phi_{n}, \psi_{n}, \eta_{n}$ are allowed to depend upon the lower scales. The approximate solution of the system of equations (1)-(9) can be obtained by using Eqs. (10)-(12) and equating the coefficients of equal powers in $\varepsilon$. We obtain the linear as well as successive higher-order perturbation equations. The hierarchy of equations for each order can be solved with the knowledge of the solution of the previous order. Furthermore, it is sufficient to consider only the solutions up to $O\left(\varepsilon^{4}\right)$ as far as the lowest significant approximation is concerned.

We introduce the linear operators defined as

$$
\begin{gathered}
L_{1}=\frac{\partial^{2}}{\partial x_{0}^{2}}+\frac{\partial^{2}}{\partial z^{2}}, \quad L_{2}\left(\eta, \phi^{(j)}\right)=\frac{\partial \eta}{\partial t_{0}}-\frac{\partial \phi^{(j)}}{\partial z}, \\
L_{3}\left(\psi^{(1)}, \psi^{(2)}\right)=\mu \frac{\partial \psi^{(1)}}{\partial z}-\frac{\partial \psi^{(2)}}{\partial z}, \\
L_{4}\left(\psi^{(1)}, \psi^{(2)}, \eta\right)=\frac{\partial \psi^{(1)}}{\partial x_{0}}-\frac{\partial \psi^{(2)}}{\partial x_{0}}+\frac{(\mu-1)}{\mu} H \frac{\partial \eta}{\partial x_{0}}, \\
L_{5}\left(\phi^{(1)}, \phi^{(2)}, \eta, \psi^{(1)}\right)=\rho_{1} \frac{\partial \phi^{(1)}}{\partial t_{0}}-\rho_{2} \frac{\partial \phi^{(2)}}{\partial t_{0}}+\left(\rho_{1}-\rho_{2}\right) g \eta \\
-T \frac{\partial^{2} \eta}{\partial x_{0}^{2}}+\frac{\mu_{2}(\mu-1)}{4 \pi} H \frac{\partial \psi^{(1)}}{\partial z} .
\end{gathered}
$$

The second-order problem $O\left(\varepsilon^{2}\right)$ is governed by the equations

$$
L_{1}\left(\phi_{2}^{(j)}\right)=0, \quad L_{1}\left(\psi_{2}^{(j)}\right)=0 \quad(j=1,2)
$$

with the following boundary conditions at $z=0$ :

$$
\begin{gathered}
L_{2}\left(\eta_{2}, \phi_{2}^{(j)}\right)=0, \quad L_{3}\left(\psi_{2}^{(1)}, \psi_{2}^{(2)}\right)=0, \\
L_{4}\left(\psi_{2}^{(1)}, \psi_{2}^{(2)}, \eta_{2}\right)=0, \quad L_{5}\left(\phi_{2}^{(1)}, \phi_{2}^{(2)}, \eta_{2}, \psi_{2}^{(1)}\right)=0 .
\end{gathered}
$$

The third-order problem $O\left(\varepsilon^{3}\right)$ is given by

$$
\begin{aligned}
& L_{1}\left(\phi_{3}^{(j)}\right)=-2 \frac{\partial^{2} \phi_{2}^{(j)}}{\partial x_{0} \partial x_{1}}, \\
& L_{1}\left(\psi_{3}^{(j)}\right)=-2 \frac{\partial^{2} \psi_{2}^{(j)}}{\partial x_{0} \partial x_{1}}
\end{aligned}
$$


and the boundary conditions at $z=0$ as

$$
\begin{aligned}
L_{2}\left(\eta_{3}, \phi_{3}^{(j)}\right) & =-\frac{\partial \eta_{2}}{\partial t_{1}}, \\
L_{3}\left(\psi_{3}^{(1)}, \psi_{3}^{(2)}\right) & =0 \\
L_{4}\left(\psi_{3}^{(1)}, \psi_{3}^{(2)}, \eta_{3}\right) & =\left(\frac{\partial \eta_{1}}{\partial x_{1}}\right) \frac{H}{\mu}(1-\mu)-\left(\frac{\partial \psi_{2}^{(1)}}{\partial x_{1}}-\frac{\partial \psi_{2}^{(2)}}{\partial x_{1}}\right), \\
L_{5}\left(\phi_{3}^{(1)}, \phi_{3}^{(2)}, \eta_{3}, \psi_{3}^{(1)}\right) & =-\rho_{1} \frac{\partial \phi_{2}^{(1)}}{\partial t_{1}}+\rho_{2} \frac{\partial \phi_{2}^{(2)}}{\partial t_{1}}+2 T \frac{\partial^{2} \eta_{2}}{\partial x_{0} \partial x_{1}} .
\end{aligned}
$$

For the fourth-order problem $O\left(\varepsilon^{4}\right)$, we have

$$
L_{1}\left(\phi_{4}^{(j)}\right)=-2 \frac{\partial^{2} \phi_{2}^{(j)}}{\partial x_{0} \partial x_{2}}-2 \frac{\partial^{2} \phi_{2}^{(j)}}{\partial x_{0} \partial x_{1}}-\frac{\partial^{2} \phi_{2}^{(j)}}{\partial x_{1}^{2}}
$$

and similar equations for $\psi_{4}^{(j)}$. The boundary conditions at $z=0$ now are

$$
\begin{gathered}
L_{2}\left(\eta_{4}, \phi_{4}^{(j)}\right)=-\frac{\partial \eta_{2}}{\partial t_{2}}-\frac{\partial \eta_{3}}{\partial t_{1}}+\eta_{2} \frac{\partial^{2} \phi_{2}^{(1)}}{\partial z^{2}}-\frac{\partial \phi_{2}^{(j)}}{\partial x_{0}} \frac{\partial \eta_{2}}{\partial x_{0}} \\
L_{3}\left(\psi_{4}^{(1)}, \psi_{4}^{(2)}\right)=-\eta_{2}\left(\mu \frac{\partial^{2} \psi_{2}^{(1)}}{\partial z^{2}}-\frac{\partial^{2} \psi_{2}^{(2)}}{\partial z^{2}}\right)+\left(\frac{\partial \eta_{2}}{\partial x_{0}}\right)\left(\mu \frac{\partial \psi_{2}^{(1)}}{\partial x_{0}}-\frac{\partial \psi_{2}^{(2)}}{\partial x_{0}}\right) \\
L_{4}\left(\psi_{4}^{(1)}, \psi_{4}^{(2)}, \eta_{4}\right) \\
=-\eta_{2}\left(\frac{\partial^{2} \psi_{2}^{(1)}}{\partial x_{0} \partial z}-\frac{\partial^{2} \psi_{2}^{(2)}}{\partial x_{0} \partial z}\right)-\left(\frac{\partial \eta_{2}}{\partial x_{0}}\right)\left(\mu \frac{\partial \psi_{2}^{(1)}}{\partial x_{0}}-\frac{\partial \psi_{2}^{(2)}}{\partial x_{0}}\right) \\
+\left(\frac{1-\mu}{\mu}\right) H\left(\frac{\partial \eta_{2}}{\partial x_{2}}+\frac{\partial \eta_{3}}{\partial x_{1}}\right)-\left(\frac{\partial \psi_{2}^{(1)}}{\partial x_{2}}-\frac{\partial \psi_{2}^{(2)}}{\partial x_{2}}\right)-\left(\frac{\partial \psi_{3}^{(1)}}{\partial x_{1}}-\frac{\partial \psi_{3}^{(2)}}{\partial x_{1}}\right)
\end{gathered}
$$

and

$$
\begin{aligned}
L_{5}\left(\phi_{4}^{(j)},\right. & \left.\eta_{4}, \psi_{4}^{(1)}\right) \\
= & -\rho_{1} \frac{\partial \phi_{3}^{(1)}}{\partial t_{1}}+\rho_{2} \frac{\partial \phi_{3}^{(2)}}{\partial t_{1}}-\rho_{1} \frac{\partial \phi_{2}^{(1)}}{\partial t_{2}}+\rho_{2} \frac{\partial \phi_{2}^{(1)}}{\partial t_{2}} \\
& -\frac{\rho_{1}}{2}\left[\left(\frac{\partial \phi_{2}^{(1)}}{\partial x_{0}}\right)^{2}+\mu\left(\frac{\partial \phi_{2}^{(1)}}{\partial z}\right)^{2}\right]+\frac{\rho_{2}}{2}\left[\left(\frac{\partial \phi_{2}^{(2)}}{\partial x_{0}}\right)+\mu\left(\frac{\partial \phi_{2}^{(2)}}{\partial z}\right)\right] \\
& -\eta_{2} \rho_{1} \frac{\partial^{2} \phi_{2}^{(1)}}{\partial t_{0} \partial z}+\eta_{2} \rho_{2} \frac{\partial^{2} \phi_{2}^{(2)}}{\partial t_{0} \partial z}+2 T \frac{\partial^{2} \eta_{2}}{\partial x_{0} \partial x_{2}}+2 T \frac{\partial^{2} \eta_{3}}{\partial x_{0} \partial x_{1}}+T \frac{\partial^{2} \eta}{\partial x_{1}^{2}} \\
& +\mu_{2}\left(\frac{\mu-1}{8 \pi}\right)\left[\left(\frac{\partial \psi_{2}^{(1)}}{\partial x_{0}}\right)^{2}+\mu\left(\frac{\partial \psi_{2}^{(1)}}{\partial z_{0}}\right)^{2}\right. \\
& \left.+\left(\frac{\partial \eta_{2}}{\partial x_{0}}\right) \frac{H^{2}}{\mu^{2}}(1-\mu)-2 H \eta_{2} \frac{\partial^{2} \psi_{2}^{(1)}}{\partial z^{2}}+\left(\frac{\mu-1}{\mu}\right) 2 H \frac{\partial \eta_{2}}{\partial x_{0}} \frac{\partial \psi_{2}^{(1)}}{\partial x_{0}}\right] .
\end{aligned}
$$


If we neglect the second harmonic resonance, then the linear theory yields the dispersion relation $[1,3]$

$$
D(\omega, k)=-\omega^{2}\left(\rho_{1}+\rho_{2}\right)+\left(\rho_{1}-\rho_{2}\right) g k+T k^{3}-\mu_{2} \frac{(\mu-1)^{2} H^{2} k^{2}}{4 \pi \mu(\mu+1)}=0 .
$$

Here $\omega, k$ denote the frequency and the wavenumber of the fundamental mode only and $\mu=\mu_{1} / \mu_{2}$. Equation (31) furnishes the critical value of the magnetic field when

$$
\begin{gathered}
k_{\mathrm{c}}=\left[\frac{\left(\rho_{1}-\rho_{2}\right) g}{T}\right]^{1 / 2}, \\
H_{\mathrm{c}}^{2}=\frac{8 \pi \mu(\mu+1)}{\mu_{2}(\mu-1)^{2}}\left[\left(\rho_{1}-\rho_{2}\right) g T\right]^{1 / 2} .
\end{gathered}
$$

The instability sets in when $H \geq H_{\mathrm{c}}$. The system bifurcates into a new steady state for the post critical values of the magnetic field. Cowley and Rosensweig [2] have experimentally verified the value of the critical magnetic field given by Eq. (33).

It was pointed out by Malik and Singh [3] and Kant and Malik [10] that when $k^{2}=\frac{1}{2}\left[\left(\rho_{1}-\rho_{2}\right) g / T\right]$, the second-order solutions have a singularity. Physically, this represents second harmonic internal resonance. The wavenumber $k$ here is independent of the magnetic field. For ordinary surface capillary-gravity waves, the resonant wavenumber is given by $k=\left(g \rho_{1} / 2 T\right)^{1 / 2}$ and the perturbation expansions are no longer uniformly valid for the second-order problem due to nonlinear focusing (see McGoldrick [14]).

With a view to describe the second harmonic resonance on the marginally neutral curve obtained by Eq. (31), it is required that, in addition to the fundamental mode $A_{1}\left(x_{1}, x_{2} ; t_{1}, t_{2}\right)$, we must take into consideration the harmonic mode $A_{2}\left(x_{1}, x_{2} ; t_{1}, t_{2}\right)$, where $\omega_{2}=2 \omega_{1}+O(\varepsilon)$ and $k_{2}=2 k_{1}+O(\varepsilon)$.

4. Dynamical equations for interacting waves. (a) Solution to second-order problem. Equations (17)-(19) for the second-order problem admit the solution

$$
\begin{gathered}
\eta_{2}=\sum_{n=1}^{2} A_{n} \exp \left(i \theta_{n}\right)+\text { c.c. } \\
\phi_{2}^{(j)}=(-1)^{j} \sum_{n=1}^{2} i\left[\frac{\omega_{n}}{k_{n}} A_{n} \exp \left(i \theta_{n}+(-1)^{j-1} k_{n} z\right)\right]+\text { c.c. }, \quad j=1,2, \\
\psi_{2}^{(1)}=\frac{H(1-\mu)}{\mu(1+\mu)} \sum_{n=1}^{2} A_{n} \exp \left(i \theta_{n}+k_{n} z\right)+\text { c.c. } \\
\psi_{2}^{(2)}=-\frac{H(1-\mu)}{(1+\mu)} \sum_{n=1}^{2} A_{n} \exp \left(i \theta_{n}-k_{n} z\right)+\text { c.c. }
\end{gathered}
$$

where

$$
\theta_{n}=k_{n} x_{0}-\omega_{n} t_{0}
$$


Here, the dispersion relation is obtained as

$$
\omega_{n}^{2}=\frac{k_{n}}{1+\rho}\left[\rho-1+k_{n} V_{A}^{2}+k_{n}^{2}\right]
$$

where

$$
V_{A}^{2}=\frac{\mu_{2}(\mu-1)^{2} H^{2}}{4 \pi \mu(\mu+1) \rho_{2}}, \quad \rho=\frac{\rho_{1}}{\rho_{2}} .
$$

The resonant harmonic wavenumber can be derived from the dispersion relation (39) by letting $\omega_{2}=2 \omega_{1}$ and $k_{2}=2 k_{1}$. We get $k_{1}=\left[\frac{g}{2 T}\left(\rho_{1}-\rho_{2}\right)\right]^{1 / 2}=k_{\mathrm{m}}$. The strength of the magnetic field at the marginally neutral curve, therefore, is

$$
H_{\mathrm{m}}\left(k_{\mathrm{m}}\right)=H_{\mathrm{m}}\left(2 k_{\mathrm{m}}\right)=\left[\left(\left(\rho_{1}-\rho_{2}\right) g+k_{\mathrm{m}}^{2} T\right)\left(\frac{4 \pi \mu(1+\mu)}{\mu_{2} k_{\mathrm{m}}(\mu-1)^{2}}\right)\right]^{1 / 2} .
$$

The system is unstable for $H \geq H_{\mathrm{m}}$. We show that within the context of the nonlinear theory, the system bifurcates into a new unstable state. It is to be noted that the choice of scalings used in this paper implies that the nonlinear coupling due to the second harmonic resonance is expected to play a predominant role. It is anticipated that the nonlinear coupling shall play an efficient role in transferring energy from the mean flow to the system, because for a wave of a given amplitude, the growth rates are much larger in comparison to any other nonlinear processes.

(b) The third-order solutions. Introducing the solutions from Eqs. (34)-(40) into Eqs. (20)-(25), we obtain the following relations:

$$
\begin{aligned}
& L_{1}\left(\phi_{3}^{(j)}\right)=2(-1)^{j} \sum_{n=1}^{2} \omega_{n} \frac{\partial A_{n}}{\partial x_{1}} \exp \left[i \theta_{n}-(-1)^{j} k_{n} z\right]+\text { c.c. } \\
& L_{1}\left(\psi_{3}^{(1)}\right)=\frac{2 i H(1-\mu)}{\mu(1+\mu)} \sum_{n=1}^{2} k_{n} \frac{\partial A_{n}}{\partial x_{1}} \exp \left[i \theta_{n}+k_{n} z\right]+\text { c.c. } \\
& L_{1}\left(\psi_{3}^{(2)}\right)=\frac{-2 i H(1-\mu)}{(1+\mu)} \sum_{n=1}^{2} k_{n} \frac{\partial A_{n}}{\partial x_{1}} \exp \left[i \theta_{n}-k_{n} z\right]+\text { c.c. }
\end{aligned}
$$

The boundary conditions at $z=0$ are

$$
\begin{aligned}
L_{2}\left(\eta_{3}, \phi_{3}^{(j)}\right) & =-\sum_{n=1}^{2} \frac{\partial A_{n}}{\partial t_{1}} \exp (i \theta)+\text { c.c. } \\
L_{3}\left(\psi_{3}^{(1)}, \psi_{3}^{(2)}\right) & =0 \\
L_{4}\left(\psi_{3}^{(1)}, \psi_{3}^{(2)}, \eta_{3}\right) & =\frac{(1-\mu) H}{\mu} \sum_{n=1}^{2} \frac{\partial A_{n}}{\partial x_{1}} \exp (i \theta)+\text { c.c. } \\
L_{5}\left(\phi_{3}^{(1)}, \phi_{3}^{(2)}, \eta_{3}, \psi_{3}^{(1)}\right)= & i \sum_{n=1}^{2}\left[2 T k_{n} \frac{\partial A_{n}}{\partial x_{1}}+\left(\rho_{1}+\rho_{2}\right) \frac{\omega_{n}}{k_{n}} \frac{\partial A_{n}}{\partial t_{1}}\right] \exp \left(i \theta_{n}\right)+\text { c.c. }
\end{aligned}
$$


Since the homogeneous part of the third-order problem has a nontrivial solution, which is the same as the first-order problem, the inhomogeneous problem has a solution if and only if the inhomogeneous part is orthogonal to every solution of the adjoint homogeneous problem. This solvability condition yields

$$
2 \frac{\omega_{n}}{k_{n}}\left(\rho_{1}+\rho_{2}\right) \frac{\partial A_{n}}{\partial t_{1}}+2 T k_{n}-\frac{\mu_{2}(\mu-1)^{2} H^{2}}{4 \pi \mu(1+\mu)}=0, \quad n=1,2,
$$

along with the complex conjugate equations. With Eq. (48), the particular solution of the third-order problem can be written as

$$
\begin{aligned}
& \phi_{3}^{(1)}=\sum_{n=1}^{2} \frac{1}{k_{n}}\left[\frac{\partial A_{n}}{\partial t_{1}}+\frac{\omega_{n}}{k_{n}}\left(1-z k_{n}\right) \frac{\partial A_{n}}{\partial x_{1}}\right] \exp i\left(\theta_{n}+k_{n} z\right)+\text { c.c. } \\
& \phi_{3}^{(2)}=-\sum_{n=1}^{2} \frac{1}{k_{n}}\left[\frac{\partial A_{n}}{\partial t_{1}}+\frac{\omega_{n}}{k_{n}}\left(1+z k_{n}\right) \frac{\partial A_{n}}{\partial x_{1}}\right] \exp i\left(\theta_{n}-k_{n} z\right)+\text { c.c. } \\
& \psi_{3}^{(1)}=\frac{i H(1-\mu)}{\mu(1+\mu)} \sum_{n=1}^{2} \frac{1}{k_{n}}\left(1-z k_{n}\right) \frac{\partial A_{n}}{\partial x_{1}} \exp \left(i \theta_{n}\right)+\text { c.c. }, \\
& \psi_{3}^{(2)}=-\frac{i H(1-\mu)}{(1+\mu)} \sum_{n=1}^{2} \frac{1}{k_{n}}\left[1+z k_{n}\right] \frac{\partial A_{n}}{\partial x_{1}} \exp \left(i \theta_{n}\right)+\text { c.c. }
\end{aligned}
$$

The nonsecularity conditions (48) may also be expressed as

$$
\frac{\partial D\left(\omega_{n}, k_{n}\right)}{\partial \omega_{n}} \frac{\partial A_{n}}{\partial t_{1}}-\frac{\partial D\left(\omega_{n}, k_{n}\right)}{\partial k_{n}} \frac{\partial A_{n}}{\partial x_{1}}=0, \quad n=1,2
$$

where

$$
D\left(\omega_{n}, k_{n}\right)=-\frac{\omega_{n}^{2}}{k_{n}}\left(\rho_{1}+\rho_{2}\right)+T k_{n}^{2}+g\left(\rho_{1}-\rho_{2}\right)-\frac{\mu_{2}(\mu-1)^{2} H^{2} k_{n}}{4 \pi \mu(1+\mu)} .
$$

Since on the marginally neutral curve $H=H_{\mathrm{m}}$ and $k=k_{\mathrm{m}}$, we discover from Eqs. (53) and (54) that $\partial D / \partial \omega_{n}=0$ and $\partial D / \partial k_{n} \neq 0$. Thus,

$$
\frac{\partial A_{n}}{\partial x_{1}}=0, \quad n=1,2
$$

Equation (55) reveals that the amplitudes $A_{n}$ are independent of the faster space scale $x_{1}$, but may depend upon $x_{2}, t_{1}$, and $t_{2}$.

(c) Fourth-order solutions. We now substitute the second- and third-order solutions into the fourth-order equations (26)-(30). Ignoring nonsecular terms like 
$\exp \left( \pm 3 i \theta_{n}\right)$ and $\exp \left( \pm 4 i \theta_{n}\right)$, which are of no interest, we obtain

$$
\begin{aligned}
& L_{1}\left(\phi_{4}^{(j)}\right)=\sum_{n=1}^{2}\left[-2 \omega_{n} \frac{\partial A_{n}}{\partial x_{2}}+i \frac{\omega_{n}}{k_{n}}\left(2 z k_{n}+(-1)^{(j)}\right) \frac{\partial^{2} A_{n}}{\partial x_{1}^{2}}\right] \\
& \cdot \exp \left[i \theta_{n}-(-1)^{j} k_{n} z\right]+\text { c.c. , } \\
& L_{1}\left(\psi_{4}^{(j)}\right)=\frac{i H(1-\mu)}{(1+\mu)}(-1)^{j} \sum_{n=1}^{2}\left[2 k_{n} \frac{\partial A_{n}}{\partial x_{2}}+\left(2 z k_{n}+(-1)^{j}\right) \frac{\partial^{2} A_{n}}{\partial x_{1}^{2}}\right] \\
& \cdot \exp \left[i \theta_{n}-(-1)^{j} k_{n} z\right]+\text { c.c. , } \\
& L_{2}\left(\eta_{4}, \phi_{4}^{(j)}\right)=-\sum_{n=1}^{2} \frac{\partial A_{n}}{\partial t_{2}} \exp \left(i \theta_{n}\right)+\text { c.c. }, \\
& L_{3}\left(\psi_{4}^{(1)}, \psi_{4}^{(2)}\right)=\frac{-2 k_{1}^{2} H(1-\mu)}{(1+\mu)}\left[A_{2} \bar{A}_{1} \exp \left(i \theta_{1}\right)+2 A_{1}^{2} \exp \left(2 i \theta_{1}\right)\right] \\
& L_{4}\left(\psi_{4}^{(1)}, \psi_{4}^{(2)}, \eta_{4}\right)=-\frac{i k_{1}^{2} H^{2}(1-\mu)^{2}}{\mu(1+\mu)}\left[3 A_{2} \bar{A}_{1} \exp \left(i \theta_{1}\right)+2 A_{1}^{2} \exp \left(2 i \theta_{1}\right)\right] \\
& +\left(\frac{1-\mu}{\mu}\right) H \frac{\partial A_{n}}{\partial x_{2}} \exp \left(i \theta_{n}\right) \text {, } \\
& L_{5}\left(\phi_{4}^{(1)}, \phi_{4}^{(2)}, \psi_{4}^{(1)}, \eta_{4}\right)=\frac{k_{1}^{2} H^{2}(1-\mu)^{2} \mu_{2}}{4 \pi(1+\mu)^{2} \mu} \\
& \cdot\left[(3 \mu+1) A_{1}^{2} \exp \left(2 i \theta_{1}\right)+A_{2} \bar{A}_{1} \exp \left(i \theta_{1}\right)\right] \text {. }
\end{aligned}
$$

Proceeding as before, the fourth-order solutions are uniformly valid subject to the conditions:

$$
\begin{gathered}
\frac{\partial^{2} A_{1}}{\partial t_{1}^{2}}+\frac{\left[\partial D / \partial k_{1}\right]_{k_{1}=k_{\mathrm{m}}}}{\left(\rho_{1}+\rho_{2}\right)} \frac{\partial A_{1}}{\partial x_{2}}=k_{\mathrm{m}}^{2} \sigma\left(\frac{H^{2}}{H_{\mathrm{m}}^{2}}-1\right) A_{1}+J \sigma A_{2} \bar{A}_{1} \exp (i \Gamma) \\
\frac{\partial^{2} A_{2}}{\partial t_{1}^{2}}+\frac{i\left[\partial D / \partial k_{2}\right]_{k_{2}=k_{\mathrm{m}}}}{\left(\rho_{1}+\rho_{2}\right)} \frac{\partial A_{2}}{\partial x_{2}}=4 k_{\mathrm{m}}^{2} \sigma\left(\frac{H^{2}}{H_{\mathrm{m}}^{2}}-1\right) A_{2}+J \sigma A_{1}^{2} \exp (-i \Gamma), \\
\sigma=\frac{\mu_{2}(1-\mu)^{2}}{4 \pi \mu(1+\mu)\left(\rho_{1}+\rho_{2}\right)}, \quad \Gamma=\theta_{2}-2 \theta_{1}, \\
J=\frac{2 k_{\mathrm{m}}^{3}(\mu-1) H^{2}}{(1+\mu)} .
\end{gathered}
$$


It is not possible, in general, to solve the dynamical equations (62) and (63). For simplicity, we neglect the space dependence due to variable $x_{2}$, and consider the case of perfect resonance, i.e., $\Gamma=0$. We normalize all the physical quantities appearing in Eqs. (62) and (63) with respect to the characteristic length $\left(T / \rho_{2} g\right)^{1 / 2}$ and time $\left(T / \rho_{2} g^{3}\right)^{1 / 4}$. The dynamical equations are integrated numerically by using the fourth-order Runge-Kutta method. We consider the ferrofluid with density $\rho_{1}=$ $1.863 \times 792 \mathrm{~kg} \mathrm{~m}^{-3}$ and the air with density of $1.24 \mathrm{~kg} \mathrm{~m}^{-3}$ as an interface. This is the same system as taken by Cowley and Rosensweig [2] in their experiments on normal field instability. We have taken the initial conditions $\operatorname{Re}\left(A_{1}\right)=1, I_{\mathrm{m}}\left(A_{1}\right)=$ $0.5, \operatorname{Re}\left(A_{2}\right)=-0.5$, and $I_{\mathrm{m}}\left(A_{2}\right)=-1.5$. The initial speeds of $A_{1}$ and $A_{2}$ are taken to be zero. By taking different sets of initial data, a similar behaviour is observed. The various Figures 1-6 exhibit the plots of the amplitudes $\left|A_{1}\right|$ and $\left|A_{2}\right|$ against time. The plots 1 to 4 are drawn by taking $\mu=3.0$ and varying $\Delta\left(=\left(H^{2} / H_{\mathrm{m}}^{2}-1\right)\right)$.

Figures 5 and 6 are plotted by keeping $\Delta=0.2$ and varying the ratio of permeabilities $\mu$. All these curves depict that the amplitudes $\left|A_{1}\right|$ and $\left|A_{2}\right|$ diverge at finite time, thus leading to an explosive type of instability at the second harmonic resonance.

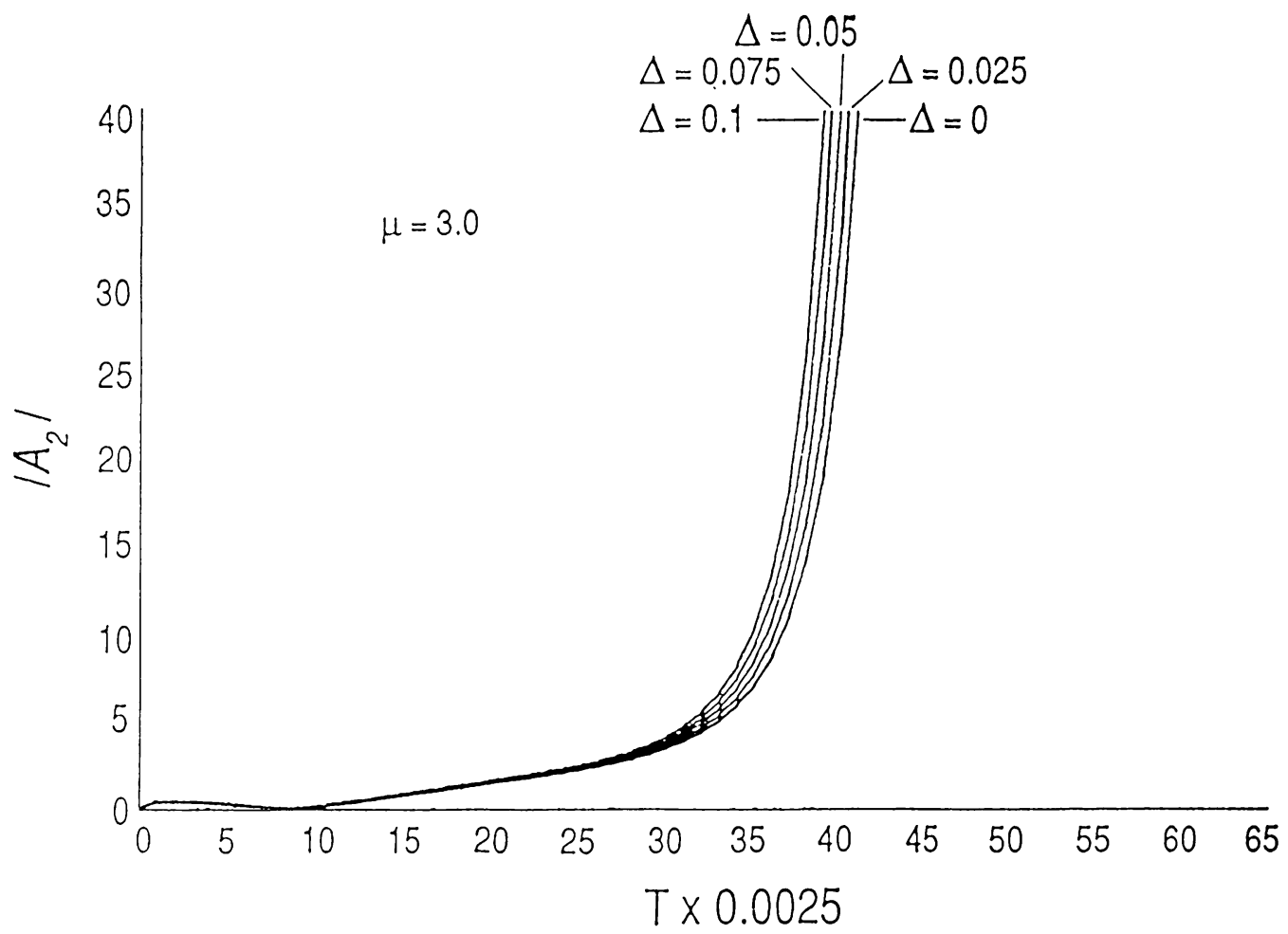

Figure 1. A plot of the fundamental model $\left|A_{1}\right|$ against time $\mu=$ 3.0 and $\Delta=0,0.025,0.05,0.075,0.1$ 


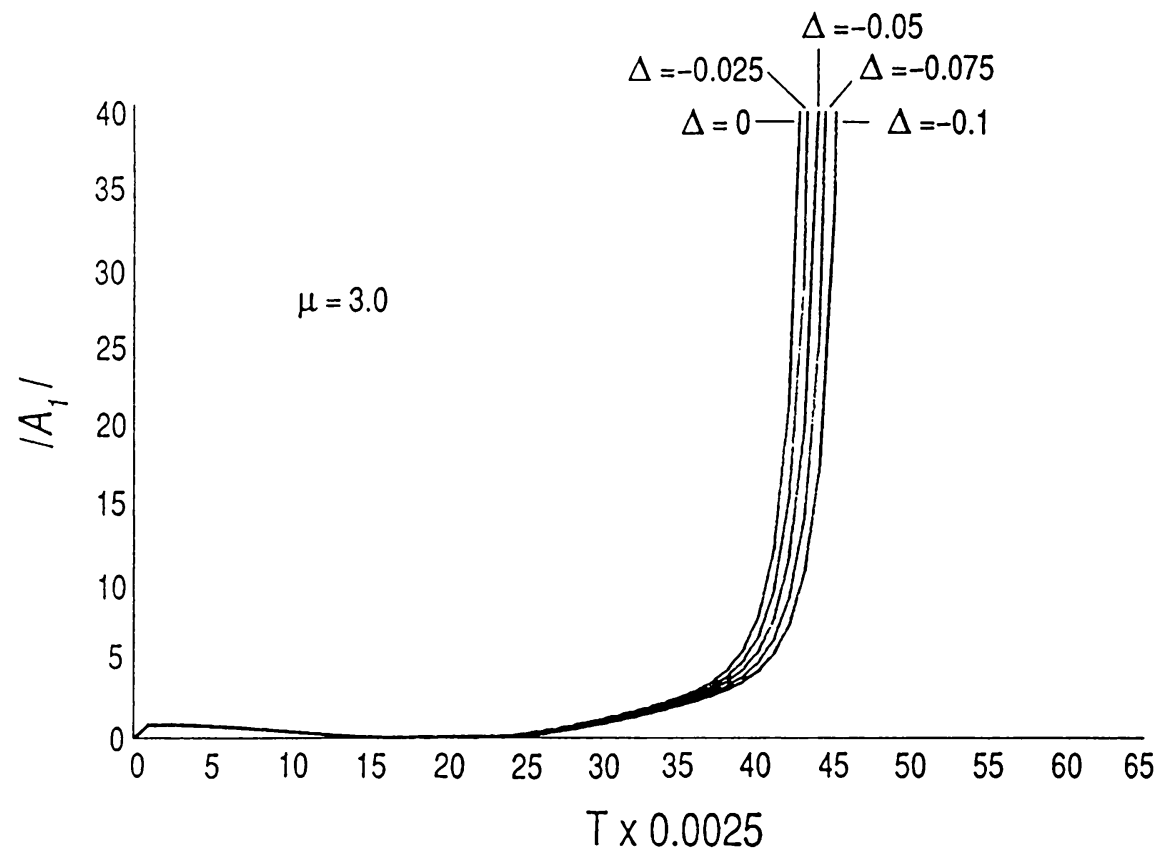

Figure 2. A plot of the fundamental mode $\left|A_{1}\right|$ against time for $\mu=3.0$ and $\Delta=0,-0.025,-0.05,-0.075,-0.01$

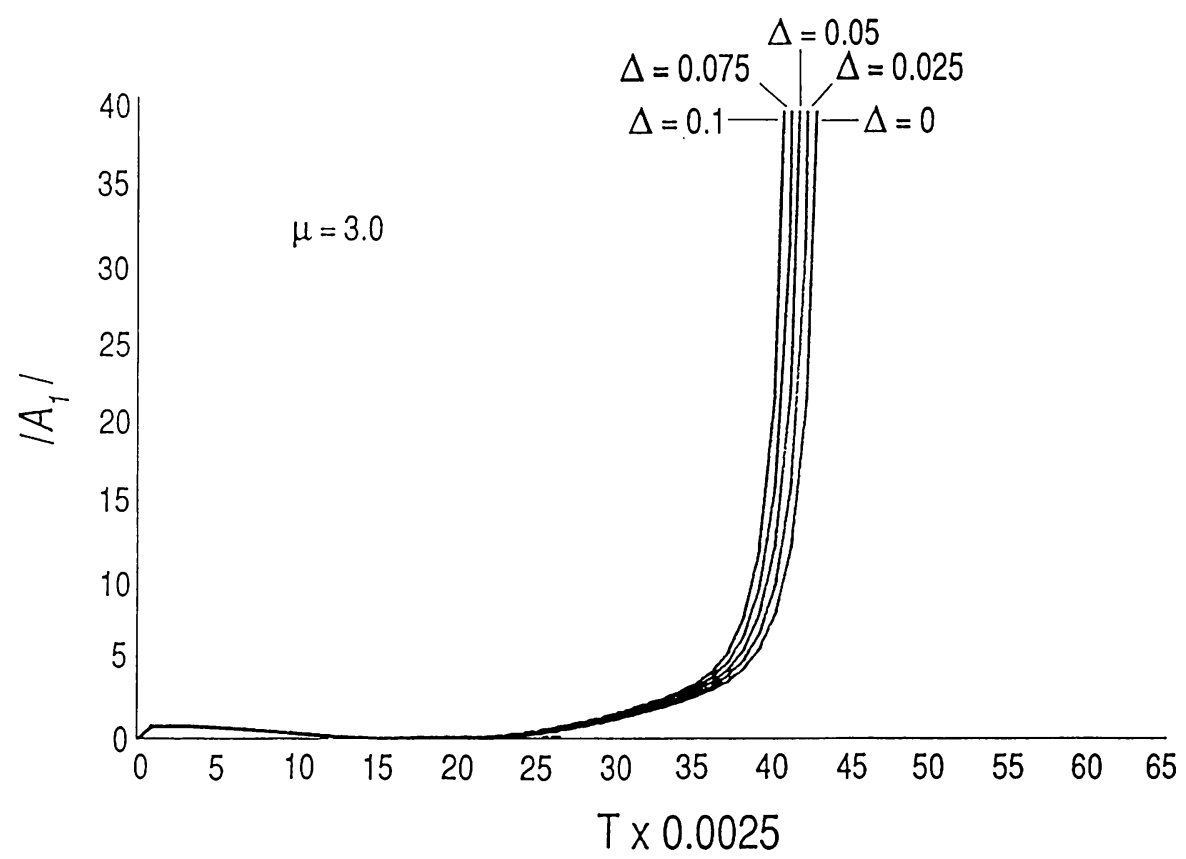

Figure 3. A plot of the second harmonic mode $\left|A_{2}\right|$ against time for $\mu=3.0$ and $\Delta=0,0.025,0.05,0.075,0.1$ 


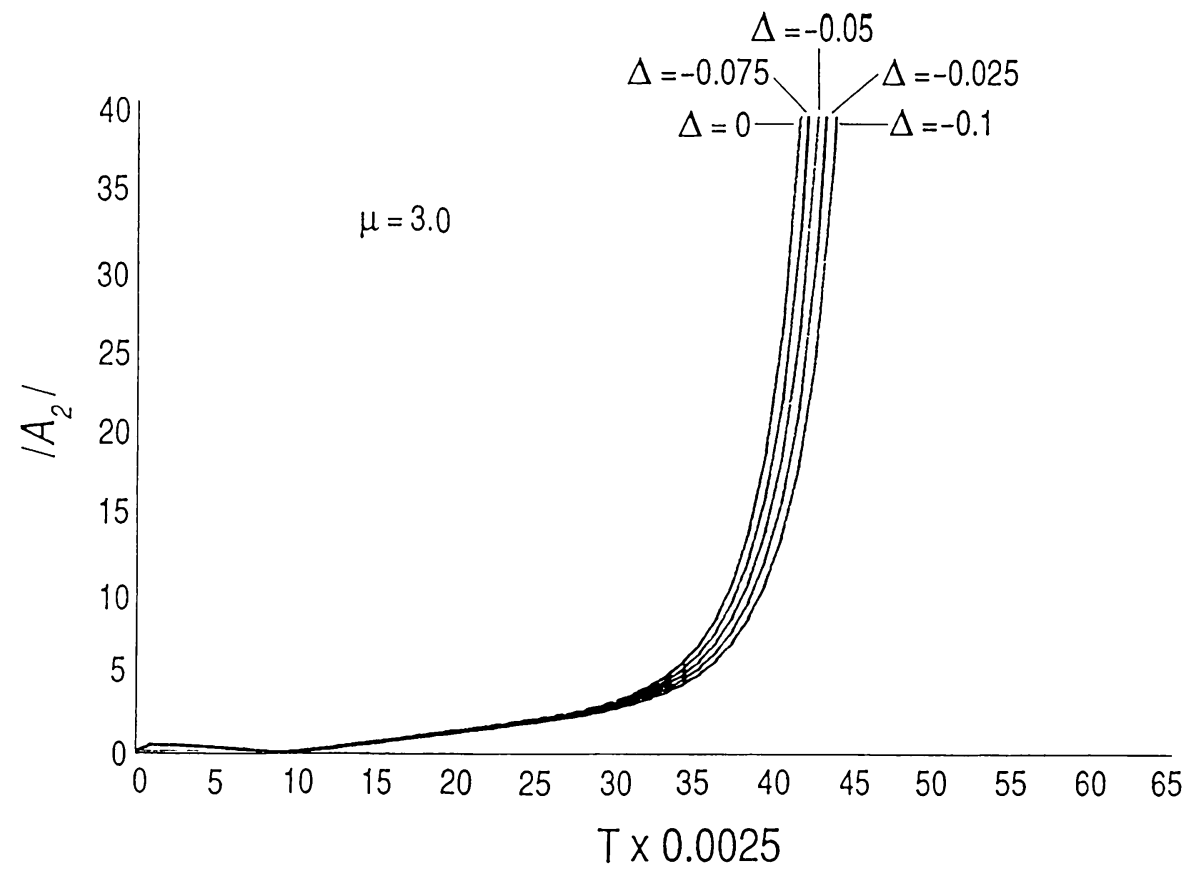

FIgURE 4. A plot of the second harmonic mode $\left|A_{2}\right|$ against time for $\mu=3.0$ and $\Delta=0,-0.025,-0.05,-0.075,-0.1$

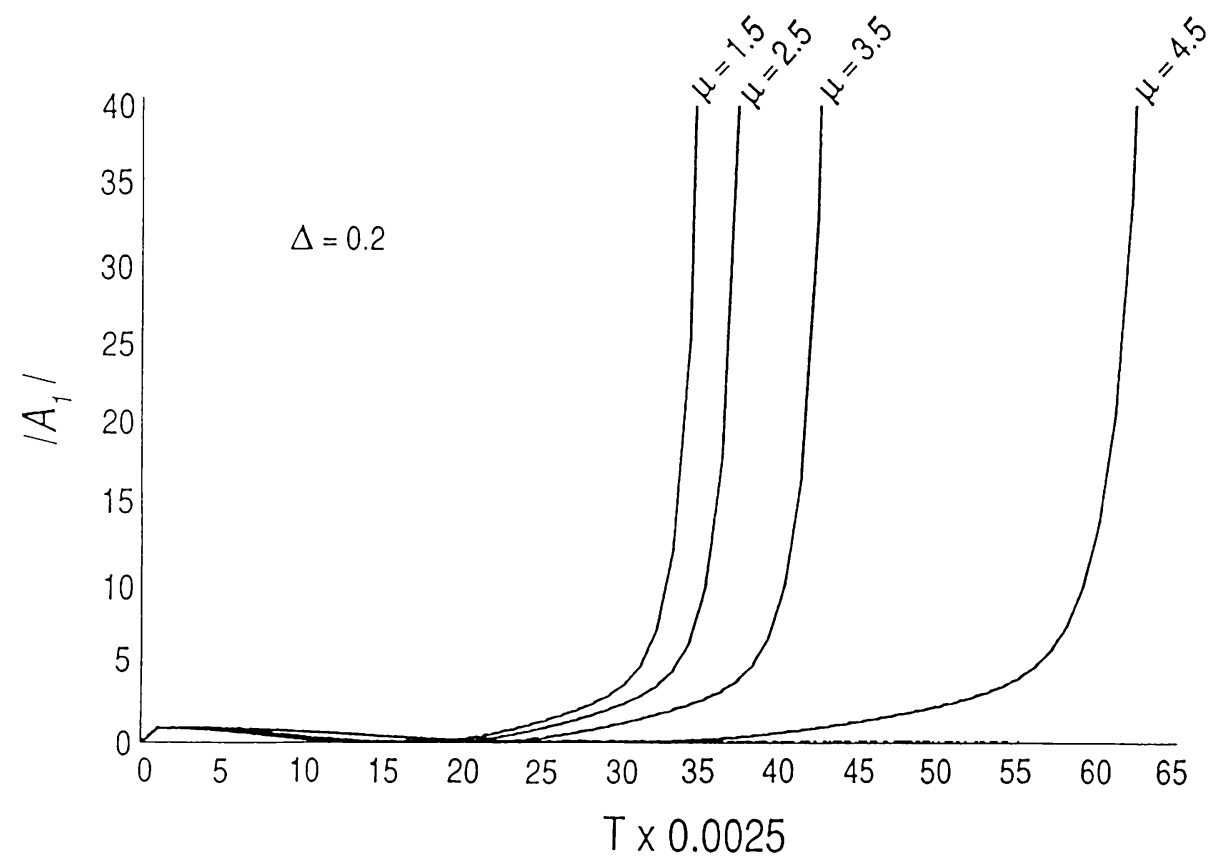

FIGURE 5. A plot of the amplitude $\left|\cdot A_{1}\right|$ against time for $\Delta=0.2$ and $\mu=1.5,2.5,3.5,4.0$ 


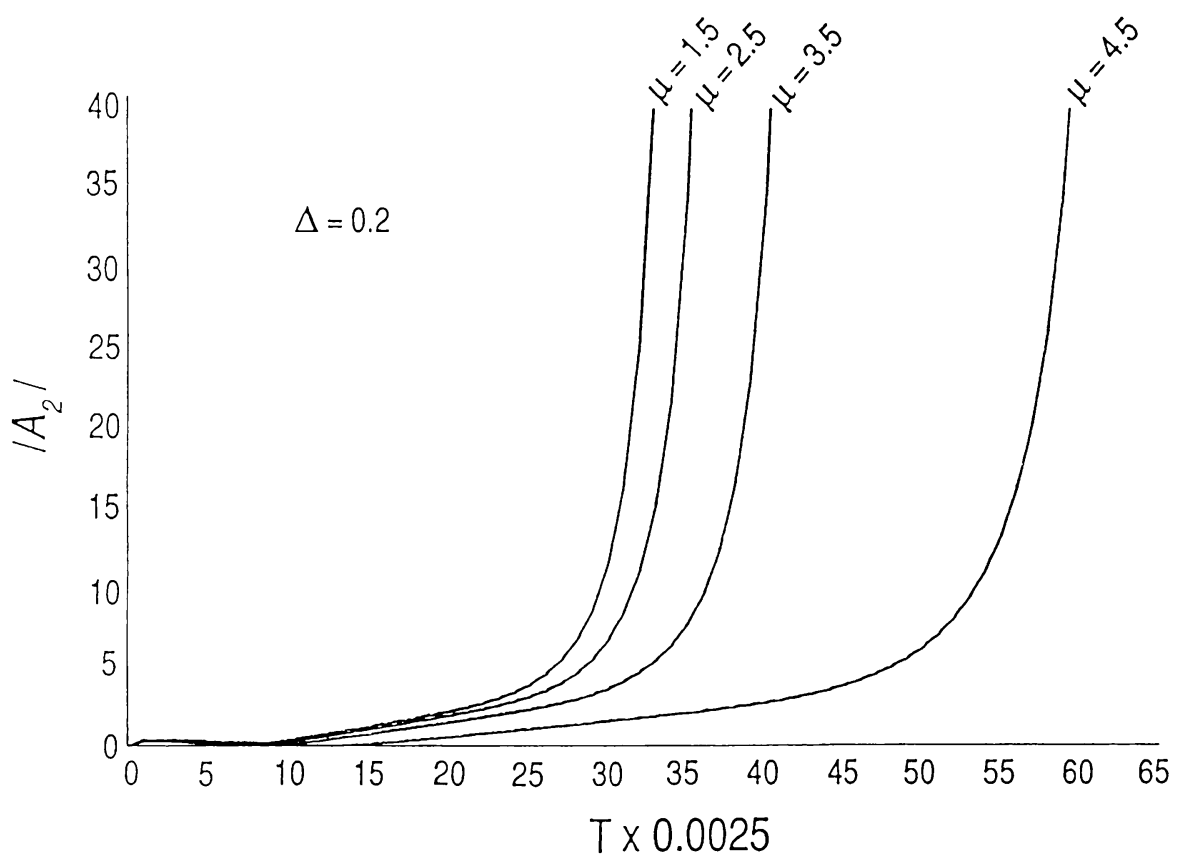

Figure 6. A plot of the amplitude $\left|A_{2}\right|$ against time for $\Delta=0.2$ and $\mu=1.5,2.5,3.5,4.0$

5. Conclusions. For a given value of $\mu$, the threshold time $\tau_{B}$ at which the amplitudes $\left|A_{1}\right|$ and $\left|A_{2}\right|$ become divergent increases as $\Delta$ goes from positive to negative values. Therefore, for the supercritical values of $H$, the explosive instability appears earlier than that for the subcritical values of $H$. The amplitude $\left|A_{2}\right|$ diverges sooner than the fundamental mode amplitude $\left|A_{1}\right|$. Figures 5 and 6 show that as $\mu$ increases, so does the time $\tau_{B}$, implying thereby stabilizing influence for the magnetic fluids of higher permeabilities. The appearance of divergent behaviour shows that when $\tau=\tau_{B}$, the perturbation procedure described in this paper becomes invalid. However, in actual practice, the explosive instability will be inhibited by the dissipating mechanism such as the viscosity of the magnetic fluids.

\section{REFERENCES}

[1] R. E. Rosensweig, Ferrohydrodynamics, Cambridge Univ. Press, 1985

[2] M. D. Cowley and R. E. Rosensweig. The interfacial stability of a ferromagnetic fluid. J. Fluid Mech. 30, 671 (1967)

[3] S. K. Malik and M. Singh, Nonlinear dispersive instabilities in magnetic fluids, Quart. Appl. Math. XLII, 359-371 (1984)

[4] A. Gailitis, Formation of the hexagonal pattern on the surface of a ferromagnetic fluid in an applied magnetic field, J. Fluid Mech. 82, 401 (1977)

[5] E. E. Trombly and J. W. Thomas, Bifurcating instabilities of the free surface of a ferrofluid, SIAM J. Math. Anal. 14, 736 (1983)

[6] J. P. Brancher, Waves and instabilities on a plane interface between ferrofluids and nonmagnetic fluids, Thermomechanics of Magnetic Fluids (ed., B. Berkovsky), Hemisphere, Bristol, PA, 1978

[7] A. G. Boudouvis, J. L. Puchalla, L. E. Scriven, and R. E. Rosensweig, Normal field instability and pattern in pool of ferrofluid, J. Magn. Magn. Mat. 65. 307 (1987)

[8] M. Silber and E. Knobloch, Pattern selection in ferrofluids. Phys. D 30, 83 (1988) 
[9] S. K. Malik and M. Singh, Nonlinear waves in magnetic fluids, Continuum Mechanics and its Applications (eds., G. A. C. Graham and S. K. Malik), Hemisphere, Bristol, PA, 1989

[10] R. Kant and S. K. Malik, Nonlinear waves in superposed magnetic fluids, Phys. Fluids 28, 35343537 (1985)

[11] R. Kant and S. K. Malik, Nonlinear internal resonance in magnetic fluids, J. Magn. Magn. Mat. 65, 347 (1987)

[12] M. A. Weissman, Nonlinear wave-packets in the Kelvin-Helmholtz instability, Philos. Trans. Roy. Soc. London Ser. A 290, 639 (1979)

[13] Y. Murakami, Second harmonic resonance on the marginally neutral curve in the Kelvin-Helmholtz flow, Phys. Lett. A 131, 368-372 (1988)

[14] L. F. McGoldrick, An experiment on second-order capillary-gravity resonant wave interactions, J. Fluid Mech. 40, 251 (1970) 